\title{
Atmospheric Correction of Landsat ETM+ Land Surface Imagery: II. Validation and Applications
}

\author{
Shunlin Liang, Senior member, IEEE, Hongliang Fang, Jeffrey T. Morisette, member, IEEE, \\ Mingzhen Chen, Chad J. Shuey, Charles L. Walthall, member, IEEE, and Craig S. T. Daughtry
}

\begin{abstract}
This is the second paper of the series on atmospheric correction of ETM+ land surface imagery. In the first paper, a new algorithm that corrects heterogeneous aerosol scattering and surface adjacency effects was presented. In this study, our objectives are to 1) evaluate the accuracy of this new atmospheric correction algorithm using ground radiometric measurements; 2) apply this algorithm to correct MODIS and SeaWiFS imagery; and 3) demonstrate how much atmospheric correction of ETM+ imagery can improve land cover classification, change detection, and broadband albedo calculations. Validation results indicate that this new algorithm can retrieve surface reflectance from ETM+ imagery accurately. All experimental cases demonstrate that this algorithm can be used for correcting both MODIS and SeaWiFS imagery. Although more tests and validation exercises are needed, it has been proven promising to correct different multispectral imagery operationally. We have also demonstrated that atmospheric correction does matter.
\end{abstract}

Index Terms-- Atmospheric correction, validation, classification, change detection, broadband albedos

\section{INTRODUCTION}

\section{【andsat TM/ETM+ images have been used extensively}

for a variety of applications [1]. Unfortunately, many images are contaminated with haze, clouds, and cloud shadows, which greatly limit their effective utilization. Moreover, quantitative retrieval of land surface information, particularly through surface reflectance models, requires the conversion from the top-of-atmosphere (TOA) radiance received by the sensor to surface reflectance.

Many efforts have been made to retrieve surface reflectance from Landsat TM imagery by correcting atmospheric effects.

Manuscript received April 15, 2002. This work was supported in part by the U.S. National Aeronautics and Space Administration (NASA) under grants NAG5-6459 and NCC5462.

S. Liang, H. Fang, M. Chen, C. Shuey are with the Department of Geography, University of Maryland, College Park, MD 20742 (telephone: 301-405-4556. email: sliang@geog.umd.edu)

M. Chen is now in the Department of Geography and Urban Planning, University of Toronto, Canada.

J. Morisette is with NASA Goddard Space Flight Center, Code 923, Greenbelt, MD 20771.

C. Walthall and C. Daughtry are with the Hydrology and Remote Sensing Laboratory, USDA ARS, Beltsville, MD 20705.
The representative algorithms have been reviewed in our previous paper [2]. One of the most popular methods is the socalled "dark-object" algorithm, which has been used for correcting imagery from several satellite systems. However, if there is no dense vegetation canopies that are evenly distributed in the scene, the "dark-object" algorithms usually fail [3]. To overcome this limitation and various disadvantages of other algorithms, we have developed a new algorithm for a general atmospheric and surface condition and therefore suitable for operational applications [2].

In the previous paper [2], we presented the major procedures and formulae, and a few correction examples were also given to demonstrate that this new method is effective for correcting ETM+ imagery under various conditions. In this paper, our first objective is to validate this algorithm quantitatively using ground radiometric measurements. The second objective is to explore the possibility of correcting other satellite images that have the similar spectral characterization, such as MODIS (Moderate-Resolution Imaging Spectroradiometer) and SeaWiFS (Sea-viewing Wide Field-of-View Sensor). The last objective is to demonstrate with practical examples how much improvement can be made for land cover classification, change detection and broadband albedo calculation with atmospheric correction.

This paper is arranged as follows: The new atmospheric correction algorithm is outlined in the next section. The validation results using ground radiometric measurements are presented in section III. The extension of this algorithm for correcting both MODIS and SeaWiFS imagery is presented in section IV. Different application examples are presented in section $\mathrm{V}$ to demonstrate the importance of atmospheric correction. A brief summary is given in the final section.

\section{The ETM+ Atmospheric Correction Algorithm}

Atmospheric effects on any satellite imagery consist of both scattering and absorption. There is no way to correct the gaseous absorption (e.g., water vapor, ozone) directly from ETM+ imagery itself unless certain ancillary information is available. If we know the surface reflectance of some pixels, for example, we might be able to estimate the gas amount of a homogeneous atmosphere. The correction procedure is straightforward if the concentrations of these gases are known. 
The major concern while we develop a new atmospheric correction algorithm is to correct scattering effects, primarily from aerosols and thin clouds, using ETM+ imagery alone.

The basic idea of this new atmospheric correction algorithm for ETM+ imagery presented in our first paper [2] is to match histograms of each cover type between the clear and hazy regions in the first three visible bands (1,2 and 3) where atmospheric scattering dominates. Each cover type is determined from the clustering analysis using three nearinfrared and middle-infrared bands (TM/ETM+ bands 4, 5 and 7) in which aerosol scattering is usually relatively low. If the haze is indeed severe or there exist some thin clouds in the image, these three bands are also affected and a histogram matching is performed for these three bands before the clustering analysis is performed.

Because of the high spatial resolution of ETM+ imagery, the surface adjacency effect is also taken into account in the atmospheric correction algorithm. An analytical formula for accounting for the surface adjacency effects was developed based on extensive simulations using a three-dimensional radiative transfer model [4].

Given aerosol optical depth and other ancillary information, surface reflectance can be retrieved by searching the look-up tables that were created by MODTRAN version 4 .

\section{VALIDATION RESUlts}

The validation site is located northeast of Washington DC covering NASA/GSFC (Goddard Space Flight Center) and USDA (U.S. Department of Agriculture) BARC (Beltsville Agricultural Research Center), an area of diverse soils, crops, and natural vegetation cover. The central point is at $\left(39.03^{\circ} \mathrm{N}\right.$, $76.85^{\circ} \mathrm{W}$ ). We are validating a series of satellite products, including the NASA MODIS (Moderate-Resolution Imaging Spectroradiometer) and MISR (Multi-angle Imaging Spectroradiometer) BRDF (Bidirectional Reflectance Distribution Function) and albedo products, and the NASA Earth Observer (EO) 1 products. Several other projects (e.g., USDA/BARC precision farming and canopy biochemistry, high-resolution IKONOS data validation project through the NASA science data buy program) are also conducting the field experiments jointly over this site. There are Sunphotometers in this site as part of the AERONET [5] that measure the aerosol optical depths and water vapor content of the atmosphere. This site has been identified as one of 24 NASA EOS (Earth Observing System) Land Core Validation Sites $[6,7]$.

We have collected many different ETM+ images over this validation site for validation purposes. All images are with different atmospheric conditions and atmospheric correction is necessary. During the Landsat-7 overpass on the following four dates (May 11, 2000, Oct 2, 2000, Apr 28, 2001 and Aug 2 , 2001), we conducted simultaneous ground radiometric measurements of surface reflectance spectra of a variety of cover types using the ASD (Analytical Spectral Devices) spectroradiometer (covering the spectrum of $0.35-2.5 \mu \mathrm{m}$ ). In a typical field campaign for surface reflectance measurements, we usually carried three ASD spectroradiometers at three different parts of our test site. During the period of the satellite overpass (about \pm one hour), each group with one ASD spectroradiometer measured surface reflectance over three or four fields (plots) that were visually homogenous with the typical size of 200-300 meters on each side. In each field (plot), about 50-100 points along several transactions were measured. Depending on the atmospheric conditions, a white reference panel was measured every point or every few points. The ASD spectroradiometer detector was held by hand and usually operated in radiance mode with a $22^{\circ}$ field of view pointing at the nadir direction. The readings of the target and the white panel produce reflectance, in which the reflectance of the white panel provided by the manufacturer was also considered. The average reflectance of these points was used to represent the mean reflectance of that field (plot). As a result, we can obtain about 10 surface reflectance spectra of different cover types in each field campaign around the satellite overpass time.

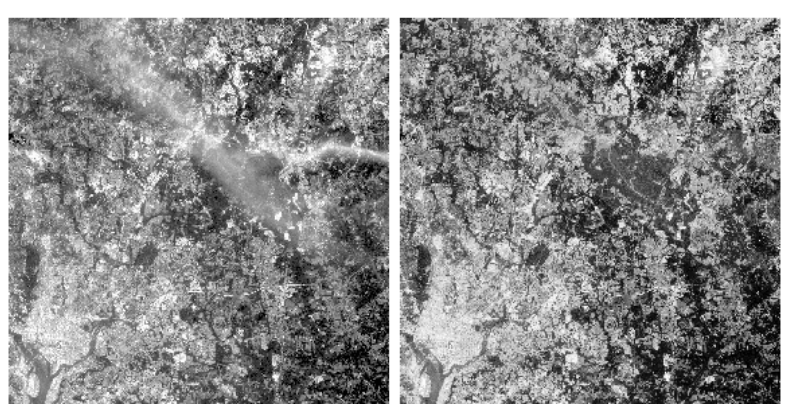

Fig.1: ETM+ band 1 imagery acquired on July 28, 1999 centered at the Beltsville, Maryland, USA, before (left) and after (right) atmospheric correction. The image size is 1500 by 1500 .

Let us first examine some examples of atmospheric correction over our test site. Figure 1 shows the differences of ETM+ band-1 imagery acquired on July 28, 1999 before and after atmospheric correction. Washington DC is in the lower left corner. There are significant improvements on the corrected image where some haze and thin clouds have been effectively removed. The image size is 1500 by 1500 .

The color composite images of bands 4,3 and 2 of May 11, 2000 before and after atmospheric correction are shown in Figure 2. The image size is 512 by 512 . A large patch of shadows has been removed and the difference is significant. The procedure for removing shadows was briefly mentioned in our first paper. Its idea is consistent with the whole correction algorithm. The near-IR bands are first used for 
identifying the shadow regions, and the visible bands are then used for classifying cover types through the clustering analysis. Reflectances of different clusters within the shadow region are then matched to those in the non-shadow regions. It is hard to see the significant difference in the same image by examining only the first band because strong scattering has masked out the shadows.
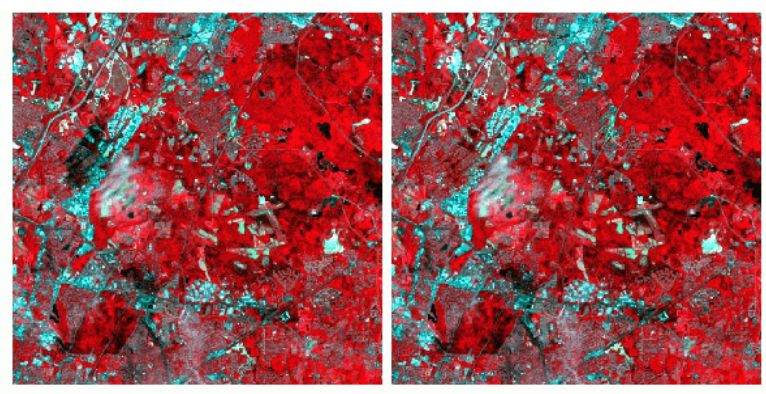

Fig.2: Color composite of ETM+ bands 4 (R),3 (G), and 2 (B) imagery acquired on May 11, 2000 centered at the Beltsville, Maryland, USA, before (left) and after (right) atmospheric correction. The image size is 512 by 512 .
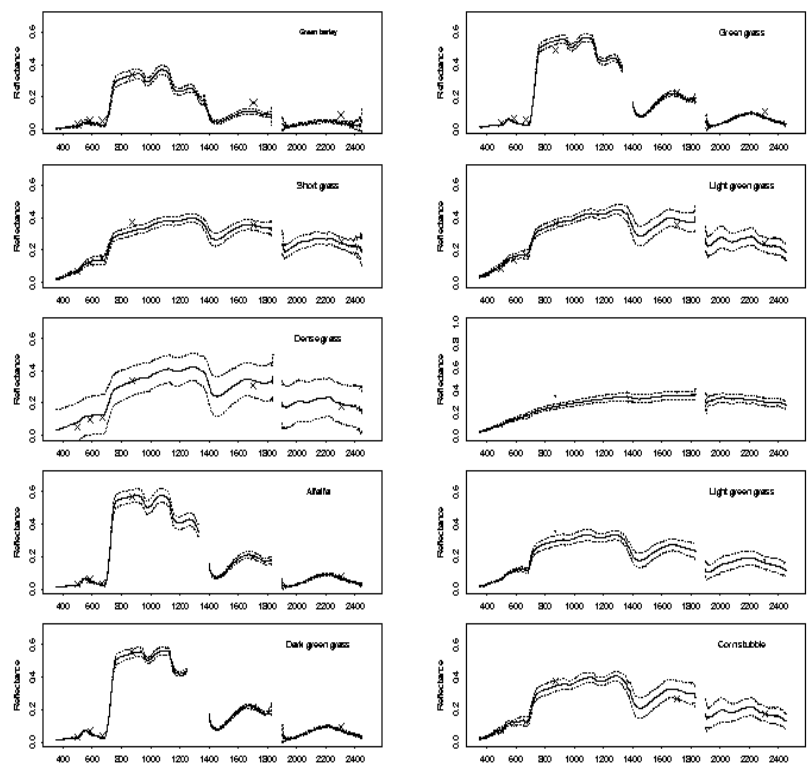

Fig.3: Comparisons of surface spectral reflectance spectra with the retrieved surface reflectance from ETM+ imagery at many different sites on April 28, 2001

Visually it seems that atmospheric correction of the July 28 , 1999 image is more effective than May 11, 2000 imagery. In both images, cloud shadows have been effectively removed. Most of the hazes, including some thin clouds, have also been well corrected. The basic assumption of the current method is that haze does not have as much impact on near-IR bands $(4,5$ and 7). If this requirement is not met, we cannot effectively remove this type of haze. For this reason some haze/clouds cannot be removed from the May 11, 2000 image.

Second, let us compare the retrieved ETM+ band reflectance from atmospheric correction with the measured reflectance spectra of different cover types at different locations. One example is given in Fig.3. The corrected pixel values represent the average reflectances of each plot and shown by symbol cross in the figure. They are compared with the average ASD measurements in these ten Sites shown by lines in the figure. The solid line is the mean reflectance, two dash lines correspond to \pm one standard deviation. The gaps indicate the absorption bands where ASD detectors do not work. From this figure, we can see that the reflectances of all these bands are well matched.

If we combine all data sets on these four dates, a much clearer picture can come out with regarding to the uncertainty of the correction procedure. Fig.4 compares the retrieved ETM+ reflectance and the aggregated ASD measurements using the ETM+ sensor spectral response functions for each band. They are not perfectly matched, but most points are distributed closely around the 1:1 line. The absolute errors in terms of the root mean squares error (RMSE) are larger in the near-IR bands from 0.027-0.041 than those in the visible bands from 0.009 to 0.015 . Since the absolute reflectance values are larger in the near-IR bands, the relative errors are quite similar, around $10 \%$. This relative error seems a little
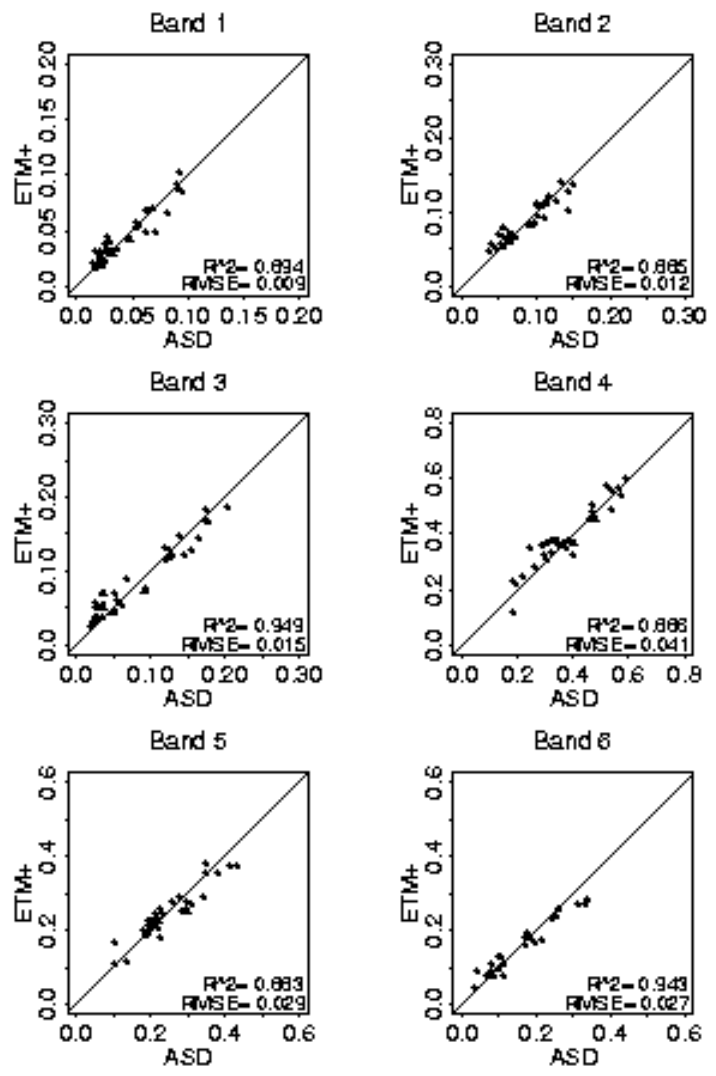

Fig4: Comparison of the retrieved ETM+ reflectance and the aggregated ASD measured reflectance acquired on four dates (May 11, 2000, Oct 2, 2000, Apr 28, 2001 and Aug 2, 2001). 
bit larger than those reported in the literature. Given the uncertainty of the aerosol scattering model, ASD measurement errors, and surface heterogeneity in our test site, we feel this is the reasonable estimate of the uncertainty in our procedure.

Another validation activity was to compare the reflectance of the "invariant" objects retrieved from imagery acquired at two different dates. We selected a set of sites whose reflectances are believed consistent at these two dates (July 28, 1999 and May 11, 2000). A total of 9 plots were selected (two conifer forest plots, two lake plots, one highway intersection plot, two plots in the Beltsville airport and two asphalt plots). The mean reflectance values of a 3 by 3 pixel window for each plot were calculated. The surface reflectance values of the first three bands from two ETM+ images are compared in Figure 5. They are in an excellent agreement. The maximum deviation is about 0.02 , but the residual standard error is 0.011 , and $R^{2}$ is 0.969 . Note that the relative errors for those points with low reflectances are nor small. These differences may be attributed to the real surface changes, registration errors or the atmospheric correction algorithm itself.

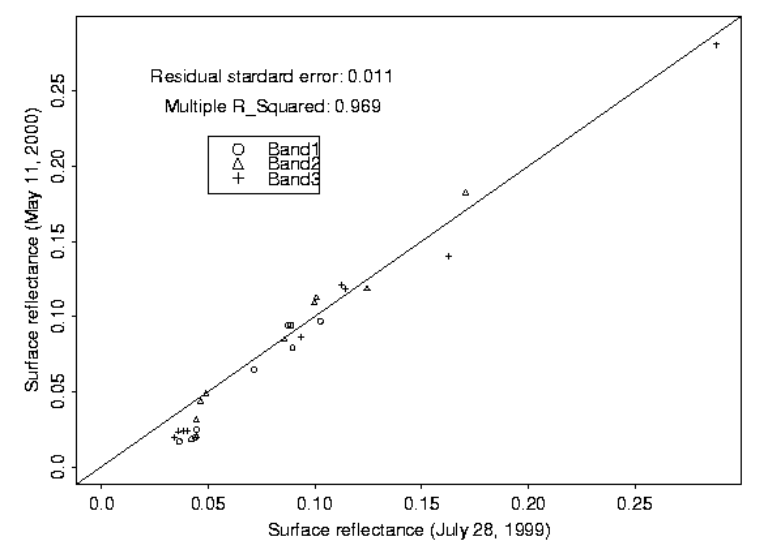

Fig.5: Comparisons of the retrieved surface reflectance from three ETM+ visible bands of May 11, 2000 and July 28, 1999 at nine "invariant" plots

Based on the direct comparison between the retrieved surface reflectance from ETM + and the simultaneously measured surface reflectance spectra, and between the retrieved surface reflectances of "invariant" objects over the two images, we can conclude that this new atmospheric correction algorithm is effective and capable of retrieving surface reflectance accurately.

\section{EXTENSIONS FOR CORRECTING OTHER SATELLITE IMAGERY}

To test the basic assumptions and principles of the ETM+ atmospheric correction algorithm, we have extended this method to correct both MODIS and SeaWiFS imagery. The extension is straightforward. The basic procedure is more or less the same - the only significant changes were the creation of different look-up tables.

\section{A. MODIS}

MODIS (Moderate-Resolution Imaging Spectroradiometer) science team is correcting the MODIS imagery operationally using the "dark object" method for estimating aerosol optical depth ([8]), but it does not work over non-vegetated surfaces. Our new method was designed for general atmospheric and surface conditions. MODIS first few bands correspond to ETM+ spectral bands closely except one more near-IR band. Their spectral characteristics are shown in Table 1. One more near-IR band enables us to identify same cover types more effectively during the clustering analysis. Below is an example with the MODIS imagery of the northeastern coast of China acquired on May 7, 2000. The image size is 400 by 400 at $1 \mathrm{~km}$ resolution. Fig. 6 compares the blue band imagery (band 3) before and after atmospheric correction, and Fig 7 compares the false color composite imagery using bands 2,1 and 4 before and after atmospheric correction. The differences are significant, particularly over the blue band. From the corrected imagery, we can tell haze or thin cloud has been effectively removed. Note that the color of the corrected imagery in figure 7 looks distorted from the original imagery mainly because of the image enhancement processing for visualization. Since we did not have any ground truth of the surface spectral reflectance, it is hard to evaluate the correction accuracy quantitatively. Besides, we did not use the MODIS cloud mask product so we are not sure whether we have removed cloud scattering effects or aerosol scattering effects since cloud and aerosol particles have different sizes and therefore different scattering effects.
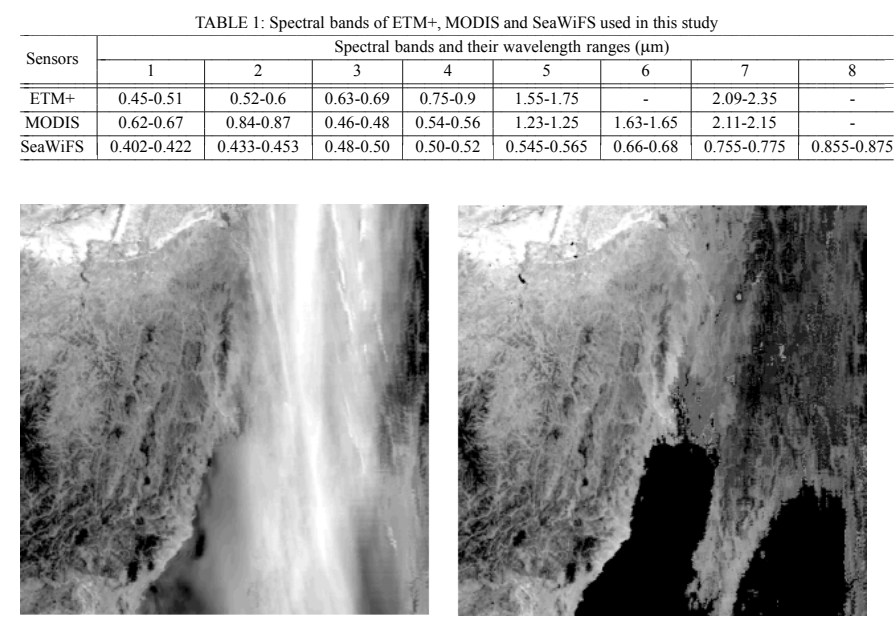

Fig. 6 MODIS Band 3 (blue) before (left) and after (right) atmospheric correction. The image size is 400 by 400 . 

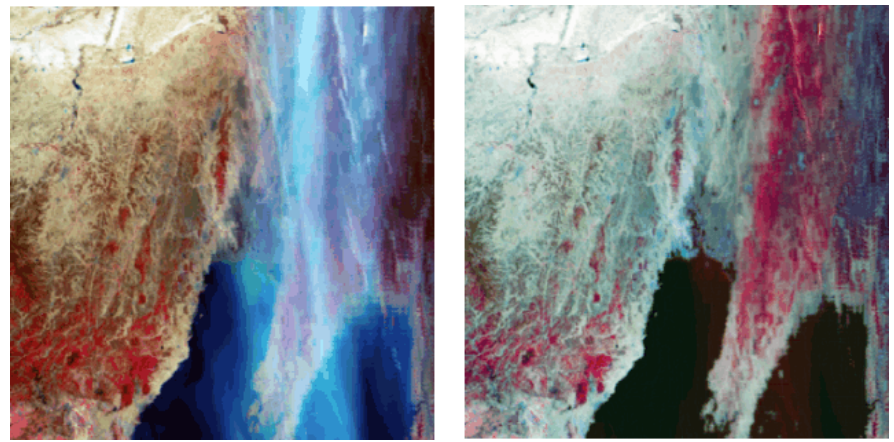

Fig. 7, MODIS false color composite imagery before (left) and after (right) atmospheric correction: RGB with bands 2 (near-IR), 1 (red) and 4 (green)

\section{B. SeaWiFS}

The SeaWiFS (Sea-viewing Wide Field-of-View Sensor) was designed for observing ocean productivity, but radiances over land are not saturated. It has total 8 bands and 6 of them are in the visible spectrum, which are highly contaminated by aerosols. Their spectral characteristics are also shown in Table 1. It has more shortwave bands, particularly one more blue band compared to $\mathrm{ETM}+$. Aerosol scattering is much stronger in blue band than in other longer wave bands. It enables us to determine the hazy regions more effectively. However, it has only two near-IR bands. Theoretically, more near-IR bands can help us identify more cover types in clustering analysis. Fortunately, SeaWIFS imagery has a much coarser spatial resolution, and the spatial variations are much smaller than ETM+ imagery. Thus, the number of clusters used in the correction procedure can be smaller and two near-IR bands seem adequate to distinguish them in the clustering analysis. Below is the image of the Washington D.C. area acquired on Nov. 6, 2000. The black/white image is band 2 (blue) and the color imagery is composed of bands 3(blue), 5(green) and 7 (near-IR). The same conclusion can be drawn from this figure: this extended algorithm can effectively remove hazes and retrieve surface reflectance.
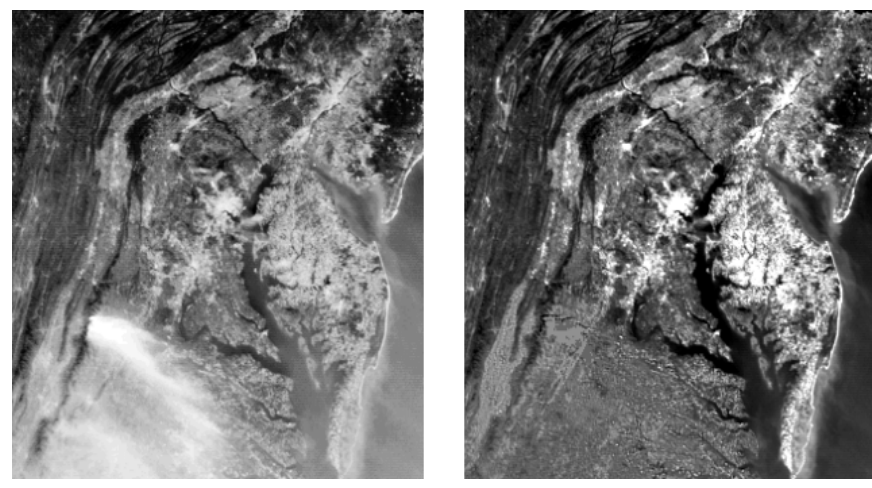

Fig. 8, SeaWiFS Band 2 (blue) before (left) and after (right) atmospheric correction
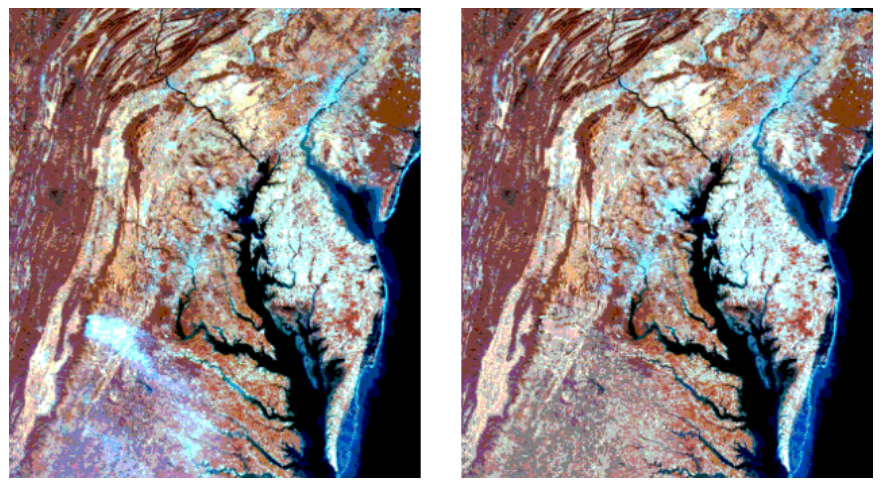

Fig. 9 the false color composite imagery using bands 7(near-IR), 5 (green) and 3(blue) for RGB. The image size is 512 by 512 .

Although it might be premature to claim that this technique is equally suitable for these low spatial resolution images, it has demonstrated that this algorithm is very promising.

\section{APPLICATIONS}

It requires great effort to retrieve surface reflectance through atmospheric correction. Also, it has often been the case in the past that atmospheric correction algorithm developments do not connect with the applications. A further step beyond developing a new algorithm is for us to demonstrate the benefits of such an effort. The values of developing a sophisticated atmospheric correction algorithm for ETM+ imagery are demonstrated by the following examples.

\section{A. Image Classification}

Land cover classification has recently been a hot research topic for a variety of applications [9]. The impacts of atmospheric correction on image classification are evaluated by Kaufman [10]. If atmospheric composition is homogeneous over the scene, it may not be necessary to conduct accurate atmospheric correction for classification purposes. When the aerosols are heterogeneously distributed over the scene, however, it may cause significant errors in image classification if no atmospheric correction is conducted. When multi-temporal images are used, atmospheric correction is highly desirable.

Figure 10 compares the classification results of the image acquired on July 28, 1999 before and after our atmospheric correction algorithm was applied. The same training sites were used in both cases. Training areas in the original, uncorrected image were identified. The criteria for selection as a training area were 1) that the area be typical of the land cover type, with many pixels representing the range of values for the cover type, and 2) The training areas also had to be in an atmospherically uncontaminated area of the image. The land cover classes were chosen based on our knowledge of the area and are: low density urban, high density urban, bare soil, 
deciduous forest, coniferous forest, and agricultural. Because of the presence of heavy haze in the upper right corner, the cover types are incorrectly classified using the original imagery before atmospheric correction. After the atmospheric correction, the cover types were correctly classified. The classification results were not thoroughly checked, however they appear to be much better based on our visits to several locations within the study area.
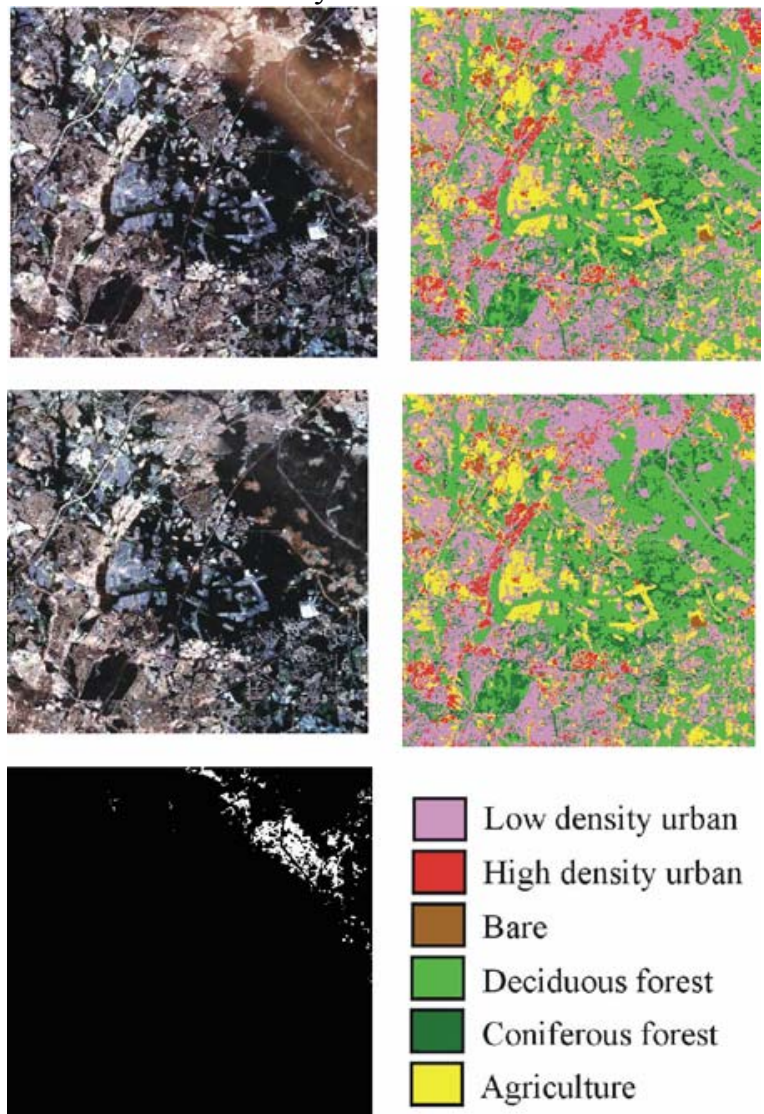

Fig.10: Evaluation of the impacts of atmospheric correction on land cover classification. The top panel is the true color composite imagery before (left) and after (right) atmospheric correction. The middle panel shows the classification results before (left) and after (right) correction. The difference mask is shown in the lower left. The lower right shows the classification legend.

\section{B. Change Detection}

Land cover change detection often relies on processing multiple images from at least two dates. Proper atmospheric correction is especially critical for multi-date, satellite-derived change detection because differences in the atmosphere for the two dates can cause either false indication of change or mask areas of real change. In order to exhibit the usefulness of our atmospheric correction algorithm with respect to change detection, we utilized a statistical modeling approach [11].

The modeling is based on a logistic regression with a binary response variable representing "change" and "no-change" areas. Combining our knowledge of the area and viewing the image data, we selected "no change" and "change" areas.
Stable areas known not to have changed were located on the image and the radiance values from the corresponding pixels were coupled with a "0" - representing no change. Similarly, areas that had changed were found on the image and radiance values for these pixels were coupled with a 1 - representing change. With this collection of $0 \mathrm{~s}$ and $1 \mathrm{~s}$, and their associated radiance values, we constructed logistic regression models.

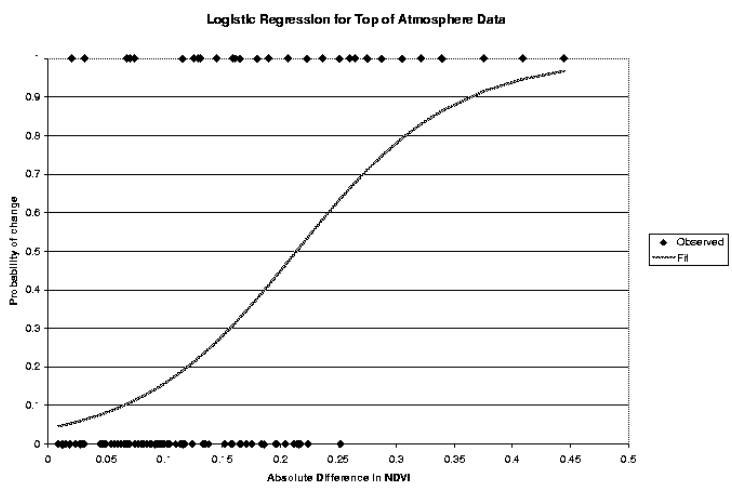

Fig. 11: Logistic regression using TOA data before atmospheric correction

For the explanatory variables we chose to use a simple absolute difference in the Normalized Difference Vegetation Index (NDVI) using ETM+ bands 3 and 4. We calculated NDVI for each date for both the TOA (top of atmosphere) reflectance and the atmospherically corrected reflectance. These will be noted as: $N D V I_{y y, r}$, where yy represents the year, either ' 99 or ' 00 and $\mathrm{r}$ represents the use of either the TOA reflectance or the atmospherically corrected (AC) reflectance.

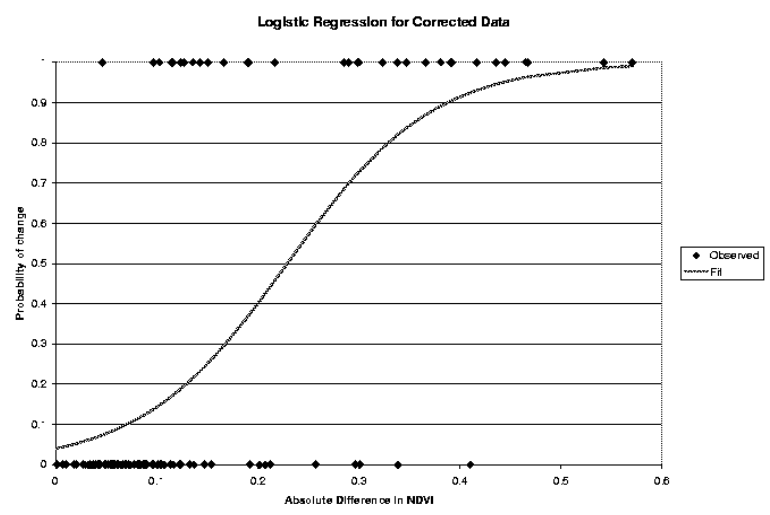

Fig. 12 Logistic regression using surface reflectance after atmospheric correction 
Then we computed the absolute difference in NDVI for the two dates:

$$
\Delta N D V I_{r}=\left|N D V I_{00, r}-N D V I_{99, r}\right|
$$

and fit two logistic regression models [12]:

$$
p(x)=\frac{e^{\alpha+\beta \Delta N D V I_{A C}}}{1+e^{\alpha+\beta \Delta N D V I_{A C}}}
$$

This allowed us to model $\mathrm{p}(\mathrm{x})$, the probability that pixel $\mathrm{x}$ has changed, by using the $0 \mathrm{~s}$ and $1 \mathrm{~s}$ representing no-change or change as the response variable and the difference in NDVI as the explanatory variable [11]. Using models (1) and (2) provides a statistical method from which to investigate the ability of the atmospherically corrected data to better distinguish between the unchanged and changed areas.

Models (1) and (2) were fit using the "Logistic" procedure in SAS. Both the observed data and fitted model are shown in Figures 11 and 12 for the $\Delta \mathrm{NDVI}_{\mathrm{TOA}}$ and $\Delta \mathrm{NDVI}_{\mathrm{AC}}$ metrics, respectively. The vertical axis represents 0 (no change) and 1 (change) for the observed data and $\mathrm{p}(\mathrm{x})$ (the modeled probability of change) for the fitted data. The horizontal axis represents the $\Delta$ NDVI metric. There appears to be more separation between the $0 \mathrm{~s}$ and $1 \mathrm{~s}$ in the observed data, with respect to their placement along the horizontal axis, for the atmospherically corrected data. This is supported more explicitly by diagnostics from the two models, which are summarized in table 2 .

Table 2: model fitting statistics

\begin{tabular}{|l|r|r|}
\hline Measure & TOA model & AC model \\
\hline AIC & 107.881 & 89.168 \\
\hline SC & 113.353 & 94.641 \\
\hline Concordance & 0.788 & 0.904 \\
\hline
\end{tabular}

Table 2 lists the Akaike Information Criterion (AIC), Schwarz Criterion (SC), and the "concordance" measure. The AIC and SC are useful for comparing different models. Better fitting models will have lower AIC and SC statistics. The concordance figure is a measure of the association between the predicted probabilities and the observed responses. A higher concordance value indicates better predictive capacity for the model. For each criterion we can see the AC model using atmospherically corrected data are superior.

From this example, we have an indication that the atmospheric correction algorithm will be helpful for change detection studies. In this study, for the sake of providing a straightforward demonstration, only NDVI values were used. Other band combinations could be used for change detection. Those using the first three bands are likely to be even more sensitive to atmospheric effects and thus the advantage of

using the corrected data would be even greater.

\section{Surface Broadband Albedo}

Land surface broadband albedo is a critical variable for many scientific applications [13]. High-resolution narrowband satellite observations contain important information that enables us to map land surface albedo globally, and validate the coarse-resolution albedo products from the broadband sensors using ground "point/plot" measurements.

There are several steps in the processing chain from the observed radiance at the top of the atmosphere to the land surface broadband albedos. The first step is to retrieve surface spectral albedos through atmospheric correction. Neglecting BRDF effects and using a nadir-only approach usually introduce errors in calculating spectral albedos. But our experiments [14] showed that these errors do not affect broadband albedos significantly. Thus, the land surface spectral albedos are approximated by the retrieved surface spectral reflectances.

The second step is to convert narrowband (spectral) albedos to broadband albedos. In a recent study, Liang [15] has developed a set of conversion coefficients based on extensive radiative transfer simulations for a variety of sensors, including ASTER (Advanced Spaceborne Thermal Emission and Reflection Radiometer), AVHRR (Advanced Very High Resolution Radiometer), ETM+/TM, GOES (Geostationary Operational Environmental Satellite), MODIS, MISR, POLDER (Polarization and Directionality of Earth's Reflectances), and VEGETATION. These formulae were found to be very effective for converting narrowband albedos to broadband albedos in a follow-up validation study [14], which compares surface albedometer measurements with simultaneous spectral measurements.
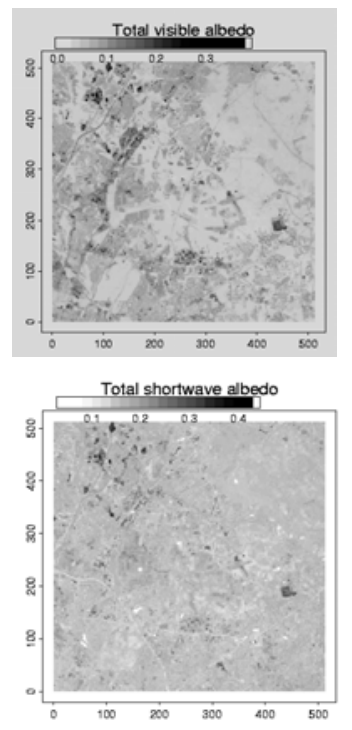

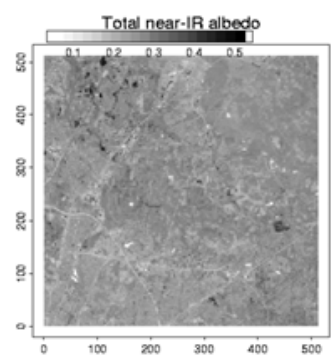

Fig. 13 Three broadband albedos retrieved from atmospherically corrected ETM+ imagery over USDA/BARC site. 
In the original article [15], coefficients are for converting ETM+/TM surface spectral reflectance to seven broadband albedos. For a quick reference, the ETM+/TM conversion coefficients for three broadband albedos (visible, near-IR and total shortwave) are given below:

$$
\left\{\begin{array}{c}
\alpha_{\text {short }}=0.356 \alpha_{1}+0.13 \alpha_{3}+0.373 \alpha_{4}+ \\
\quad 0.085 \alpha_{5}+0.072 \alpha_{7} \\
\alpha_{v i s}=0.443 \alpha_{1}+0.317 \alpha_{2}+0.24 \alpha_{3} \\
\alpha_{N I R}=0.693 \alpha_{4}+0.212 \alpha_{5}+0.116 \alpha_{7}
\end{array}\right.
$$

where $\alpha_{i}$ represents the land surface ETM+ spectral reflectance. The wavelength ranges of these three broadband regions are: total shortwave $(0.3-2.5 \mu \mathrm{m})$, visible $(0.4-0.7$ $\mu \mathrm{m})$, and near-IR $(0.7-2.5 \mu \mathrm{m})$. These three broadband albedo maps can be easily produced applying these conversion formulae to the atmospherically corrected surface reflectance. An example of these three broadband albedos from the ETM+ image acquired on July 28, 1999 is displayed in figure 13. This kind of detailed albedo map is of great importance to various applications.
(A)

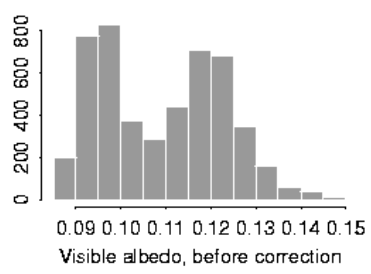

(C)

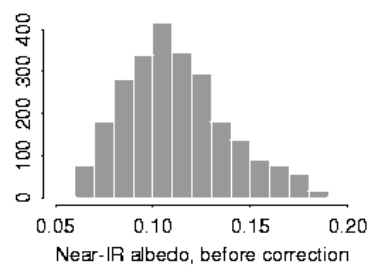

(B)

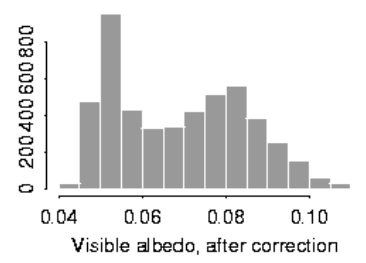

(D)

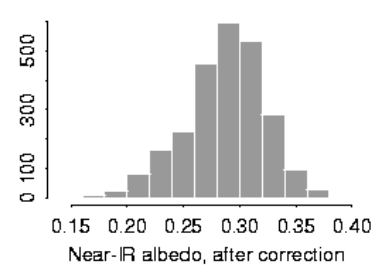

Fig.14: Histograms of visible broadband albedo from ETM+ TOA spectral reflectance (A) and from ETM+ surface spectral reflectance (B), near-IR broadband albedo from ETM+ TOA spectral reflectance (C) and from ETM+ surface spectral reflectance (D). (A) and (B) correspond to a hazy region, and (C) and (D) to a cloud shadow region

To demonstrate the impacts of atmospheric effects on land surface broadband albedo calculations, two windows were extracted. The first window of 5000 pixels is the hazy region just above the Beltsville airport in the image of July 28, 1999, and the second window of 2500 pixels is the shadowing region in the image of May 11, 2000. Broadband albedos were calculated and compared before and after atmospheric correction. The histograms of visible albedo of the first window and the histograms of near-IR albedo of the second window are shown in Figure 14. The differences are significant. The visible albedo of the hazy region is much larger (0.11 compared to corrected albedo of 0.07 ), and the near-IR albedo of the shadowing region is much smaller before atmospheric correction ( 0.11 compared to the corrected albedo of 0.29).

\section{SUMMARY}

A new atmospheric correction algorithm of ETM+ imagery was validated using simultaneous ground measurements. The results indicated that the relative differences between the retrieved surface reflectance from the corrected ETM+ imagery and surface measured spectral reflectance using a hand-held radiometer are around $10 \%$. It demonstrates that this new atmospheric correction algorithm can be used to retrieve surface reflectance accurately.

This algorithm has been extended to correct both MODIS and SeaWiFS imagery that have different spectral and spatial characteristics from ETM+ imagery. Although there were no ground truths to evaluate the accuracy of the correction algorithms, it is very clear from two examples that the corrected images were much better than the original images after large patches of haze has been removed. It illustrates that the basic assumptions used in the ETM + atmospheric correction algorithm are reasonable and it can be extended to correct many different types of satellite data.

Several applications were shown in this study to demonstrate the high values of atmospheric correction, including land cover classification, surface change detection and surface broadband albedo calculations. If haze is homogeneously distributed over the imagery, we might easily be able to correct the systematic biases due to atmospheric effects without performing a sophisticated atmospheric correction. Otherwise, atmospheric correction is always desirable.

There are several key factors that affect the accurate retrieval of land surface reflectance. The first is the total column water vapor content of the atmosphere, which significantly affect near-IR band reflectance.. Fortunately, MODIS in the EOS Terra spacecraft can produce the total water vapor content map using both water vapor absorption bands and thermal sounding bands. For the validation purpose in this paper, the measured water vapor contents were used in which a homogeneous atmosphere has been assumed

Another factor is the aerosol scattering model. In our validation site, many sensitivity experiments have shown that the accuracy of the retrieved surface reflectance is affected by the choice of the aerosol model. Fortunately, several EOS 
sensors, such as MODIS and MISR, should be able to provide us with more accurate aerosol climatology in the near future.

The validation took place only over our validation site at Beltsville, Maryland. Further work is still needed to validate this algorithm over other regions under different surface and atmospheric conditions.

\section{ACKNOWLEDGMENT}

The authors are very grateful to a group of scientists who participated in our field campaigns, including Crystal Schaaf and Feng Gao at Boston University, Peter Muller at University College London, and Ray Hunt, Andy Russ, and Wayne Dulaney at USDA Agricultural Research Center at Beltsville. We also really appreciate the access to the Sunphotometer data as part of the AERONET led by Dr. Brent Holben. We are grateful to the anonymous reviewers for their valuable comments.

\section{REFERENCES}

[1] S. N. Goward and D. L. Williams, "Landsat and Earth Systems Science: Development of terrestrial monitoring," Photogr. Eng. Remote Sens., vol. 63, pp. 887-900, 1997.

[2] S. Liang, H. Fang, and M. Chen, "Atmospheric Correction of Landsat ETM+ Land Surface Imagery: I. Methods," IEEE Transactions on Geosciences and Remote Sensing, vol. 39, pp. 2490-2498, 2001.

[3] G. Wen, S. C. Tsay, R. F. Cahalan, and L. Oreopoulos, "Path radiance technique for retrieving aerosol optical thickness over land," J. Geophys. Res., vol. 104, pp. 3132131332, 1999.

[4] K. F. Evans, "The spherical harmonics discrete ordinate method for three-dimensional atmospheric radiative transfer," J. Atmos. Sci., vol. 55, pp. 429-464, 1998.

[5] B. N. Holben, T. Eck, I. Slutsker, T. Tanre, J. Buis, A. Setzer, E. Vermote, J. Reagan, Y. Kaufman, T. Nakajima, F. Lavenu, I. Jankowiak, and A. Smirnov, "AERONET - A federated instrument network and data archive for aerosol characterization," Remote Sensing of Environment, vol. 66, pp. 1-16, 1998.

[6] C. Justice, D. Starr, D. Wickland, J. Privette, and T. Suttles, "EOS land validation coordination: An update.," Earth Observer, vol. 10, pp. 55-60, 1998.

[7] J. Morisette, J. Privette, C. Justice, D. Olson, J. Dwyer, P. Davis, D. Starr, and D. Wickland, "The EOS land validation core sites: background information and current status," The Earth Observer, vol. 11, pp. 11-26, 1999.

[8] Y. J. Kaufman, A. Wald, L. A. Lorraine, B. C. Gao, R. R. $\mathrm{Li}$, and L. Flynn, "Remote sensing of aerosol over the continents with the aid of a 2.2 um channel," IEEE Trans. Geosci. Remote Sens., vol. 35, pp. 1286-1298, 1997.

[9] J. G. R. Townshend, "Global data sets for land applications from the Advanced Very High Resolution Radiometer: an introduction," Int. J. Remote Sen., vol. 15, pp. 3319-3332, 1994.

[10] Y. Kaufman, "The atmospheric effect on separability of field classes measured from satellite," Remote Sens. Environ., vol. 18 , pp. 21-34, 1994?

[11] J. Morisette, S. Khorram, and T. Mace, " Land-cover change detection enhanced with generalized linear models," Int. J. Remote Sens., vol. 20, pp. 2703-2721, 1999.

[12] A. Agresti, Categorical Data Analysis. New York: John Wiley and Sons. 2002, $2^{\text {nd }}$ edition, pp. 710.

[13] R. E. Dickinson, "Land surface processes and climatesurface albedos and energy balance," Adv. Geophys., vol. 25, pp. 305-353, 1983.

[14] S. Liang, C. Shuey, H. Fang, A. Russ, M. Chen, C. Walthall, C. Daughtry, and R. Hunt, "Narrowband to broadband conversions of land surface albedo: II. Validation," Remote Sens. Environ., in press., 2002 b.

[15] S. Liang, "Narrowband to broadband conversions of land surface albedo," Remo Sensing of Environment, vol. 76, pp. 213-238, 2001.

Dr. Shunlin Liang received his Ph.D degree in Remote Sensing and GIS from Boston University. He was a postdoctoral research associate in Boston University from 1992 to 1993, and Validation Scientist of the NOAA/NASA Pathfinder AVHRR Land Project from 1993 to 1994. He is currently an associate professor in the University of Maryland at College Park. His present research interests focus on land surface data assimilation, parameter retrieval from remotely sensed data, and spatial analysis. He organized the International Forum on BRDF at San Francisco, December 1998, and co-edited a special issue of the Remote Sensing Reviews. He chaired various sessions of the international conferences and served as the chairman of the IEEE Geosciences and Remote Sensing Society, Washington/North Virginia Chapter (2000). He is an associate editor of the IEEE Transactions on Geoscience and Remote Sensing.

Mr. Hongliang Fang is currently a doctoral student at the University of Maryland, College Park. His previous interest was on use of remote sensing and GIS in a variety of fields such as environment monitoring, land cover/use, biomass monitoring and wetland ecology. His most recent interest is in retrieving land surface parameters from remotely sensed data.

Dr. Jeffrey T. Morisette received the B.A. degree from Siena Heights University, Adrian MI, the M.S. degree in Statistics from Oakland University, Rochester MI, and the $\mathrm{PhD}$. degree from North Carolina State University, Raleigh $\mathrm{NC}$, where he focused on geostatistics, accuracy assessment, and satellite based changed detection, funded through a 
NASA Earth System Science Graduate fellowship. He also attended the International Space University's Summer Program, Vienna, 1996.

His current research is on the application of multi-resolution satellite imagery to ecological studies and the validation of global land products with a focus on coordinating the MODIS land team validation activities through NASA's Goddard Space Flight Center. He also works with the "Land Product Validation Subgroup" of the Committee on Earth Observing Satellite Working Group on Calibration and Validation helping to organize and coordinate international land product validation activities.

Dr. Mingzhen Chen received his $\mathrm{Ph} . \mathrm{D}$ degree in remote sensing of soils from the Zhejiang Agricultural University. He was an associate professor in Zhejiang University from 1996 to 1998 . He was a visiting research scientist in the University of Maryland at College Park and currently in the University of Toronto. His research interests focus on remote sensing and GIS applications, such as retrieving land surface parameters from remotely sensed data, land cover and land use, biomass and environmental monitoring.

Mr. Chad J. Shuey is a doctoral student at the University of Maryland, College Park. He has a B.A. in Geography from George Mason University (1995), and an M.S. in Geography from the University of Akron (1997). His research interests are in map projection error and geographic visualization, as well as in the applications of remote sensing data to agriculture.

Dr. Charles L. Walthall has been involved with laboratory, field, airborne, and satellite remote sensing research since 1976. He received a B.S. in Geography from the University of Maryland in 1977, an M.S. in Forest Science with a specialization in remote sensing from Texas A\&M in 1982, and a PhD in Agricultural Meteorology and Climatology/ Agronomy with a specialization in remote sensing from the University of Nebraska in 1988. He is currently employed with the Hydrology and Remote Sensing Laboratory of the USDA Agricultural Research Service as a physical scientist where he conducts research on remote sensing applications to agriculture and natural resources. His research interests include remote sensing of vegetation and soils parameters, $\mathrm{BRDF}$, and airborne remote sensing.

\section{Dr. Craig Daughtry}

